\title{
Review of recent heavy flavor measurements in STAR
}

\author{
Michael R. Lomnitz ${ }^{1,2, a}$ (for the STAR collaboration) \\ ${ }^{1}$ Nuclear Science Division, Lawrence Berkeley National Lab, MS70R0319, One Cyclotron Road, Berkeley, \\ CA 94720, USA \\ ${ }^{2}$ Department of Physics, Kent State University, 800 E. Summit St., Kent, OH 44240, USA
}

\begin{abstract}
Heavy-ion collisions at RHIC provide a unique environment to study the behavior of nuclear matter under extreme conditions. In particular, heavy quarks, which are produced during the early stages of a collision, provide an exceptional probe in understanding the hot and dense medium created in such collisions. The Heavy Flavor Tracker and Muon Telescope Detector at the STAR experiment at RHIC have been successfully installed since early 2014 and have significantly improved the experimental capabilities in measuring both open and hidden heavy flavor hadrons in heavy-ion collisions. We present an overview of recent heavy flavor results obtained at STAR using these two dedicated detectors.
\end{abstract}

\section{Introduction}

Heavy flavor quarks are suggested to be an excellent probe to study the strongly coupled quark-gluon plasma (sQGP) as they are predominantly produced early in heavy-ion collisions through hard scattering processes. They experience the full evolution of the system, while their large masses are mostly unaffected by the QCD medium. Measurements of open heavy flavor and quarkonia at the Relativistic Heavy Ion Collider (RHIC) have revealed several interesting features such as: the enhancement (suppression) in $D^{0}$ production at intermediate (high) transverse momenta $\left(p_{T}\right)$ in $\mathrm{Au}+\mathrm{Au}$ collisions at $\sqrt{\mathrm{S}_{\mathrm{NN}}}=200 \mathrm{GeV}$ relative to $\mathrm{p}+\mathrm{p}$ collisions [1]; the weak collision energy dependence of the $J / \psi$ suppression in $\mathrm{Au}+\mathrm{Au}$ collisions from $\sqrt{\mathrm{s}_{\mathrm{NN}}}=39$ to $200 \mathrm{GeV}$ [2]. While the former can be described by theoretical frameworks including strong charm-medium interactions and charm-light quark coalescence, the latter is attributed to the interplay of color screening in the QGP and $J / \psi$ regeneration from deconfined $c \bar{c}$ pairs.

Despite significant progress in the last several decades, precise measurements are still needed to fully understand the properties of the sQGP and the parton-QGP interactions. The STAR experiment has recently completed collecting data with two new detectors designed for heavy flavor studies: the Heavy Flavor Tracker (HFT) [3] and the Muon Telescope Detector (MTD) [4]. In these proceedings we will present the first set of physics results using the HFT and the MTD.

\footnotetext{
ae-mail: mrlomnitz@lbl.gov
} 


\section{The STAR experiment}

STAR is a large acceptance, multi-purpose detector at RHIC designed to discover and study the QGP. It possesses full azimuthal coverage at mid-rapidity $(|\eta|<1)$ using the Time Projection Chamber (TPC) to reconstruct tracks inside a uniform 0.5 T magnetic field. By measuring the energy loss $(\mathrm{dE} / \mathrm{dx})$, the TPC can also provide excellent PID for charged hadrons over a broad range in $p_{T}$. Combining with information from the Time Of Flight (TOF) detector, STAR can provide accurate identification over $\sim 98 \%$ of the charged hadrons produced in heavy-ion collisions.

Since the beginning of 2014, two new detectors, the HFT and MTD, have been fully installed at STAR. The HFT consists of four layers of silicon detectors located inside the TPC. With thinned Monolithic Active Pixel Sensors (MAPS), the HFT greatly improves the track pointing resolution to be less than $50 \mu \mathrm{m}$ for kaons with $p_{T}=750 \mathrm{MeV} / \mathrm{c}$, and thus permits topological reconstruction of open heavy flavor decays through hadronic channels.

The MTD is installed outside of the STAR magnet, utilizing Multi-gap Resistive Plate Chamber (MRPC) technology. By performing precise time-of-flight measurements with resolution of $\sim 100$ ps, the MTD can trigger on and identify muons with $p_{T}>1.2 \mathrm{GeV} / \mathrm{c}$ and $|\eta|<0.5$. The HFT and MTD have collected large-statistics data sets in $\mathrm{p}+\mathrm{p}, \mathrm{p}+\mathrm{Au}, \mathrm{d}+\mathrm{Au}$, and $\mathrm{Au}+\mathrm{Au}$ collisions at $\sqrt{\mathrm{s}_{\mathrm{NN}}}=200 \mathrm{GeV}$ between 2014 and 2016. Results presented below are obtained using around 1/4 of the Minimum-Bias (MB) data with the HFT and 1/2 of the dimuon triggered data with the MTD in $\mathrm{Au}+\mathrm{Au}$ collisions.

\section{Open heavy flavor production}

The charm production cross-sections at mid-rapidity $(|y|<1)$ have been measured by STAR in $\mathrm{p}+\mathrm{p}$ and $\mathrm{Au}+\mathrm{Au}$ collisions at $\sqrt{\mathrm{s}_{\mathrm{NN}}}=200 \mathrm{GeV}$, and were found to scale with the number of binary nucleon collisions [1]. This observation supports the picture in which heavy flavor quarks are predominantly produced in initial hard parton scatterings at RHIC. Moreover, because of their relatively long lifetimes, heavy flavor quarks can experience the entire evolution of the system, serving as a calibrated probe to study the QGP. Below we present results on $D^{0}$ meson production reconstructed through the hadronic decay channels.

Using the HFT, the combinatorial background is greatly suppressed by selecting on the following topological variables: decay length (distance between primary and decay vertices), distance of closest approach (DCA) between daughter tracks, DCA between reconstructed $D^{0}$ candidate and the primary vertex (PV), and DCA between daughter tracks and the PV. Shown in figure 1a are the nuclear modification factor $\left(R_{A A}\right)$ of $D^{0}$ in $0-10 \% \mathrm{Au}+\mathrm{Au}$ collisions at $\sqrt{\mathrm{s}_{\mathrm{NN}}}=200 \mathrm{GeV}$ extracted from about 780 million MB events with the HFT (black solid circles). Also shown are the STAR published results from 1.1 billion MB events recorded in 2010 and 2011 without the HFT (red open circles) [1], as well as the $R_{A A}$ for charged pions. The vertical bars (brackets) represent statistical (systematic) uncertainties, while the grey shaded areas depict the systematic uncertainties from the $\mathrm{p}+\mathrm{p}$ reference uncertainties for the $R_{A A}$ obtained from the HFT data. The latter are not shown for the published results due to the cancellation of efficiency corrections between the $p+p$ and 2010/2011 Au+Au data, both of which had the same experimental set-up. The colored boxes centered at $R_{A A}=1$ on the far right of the panel correspond to global normalization uncertainties for 0-10\% Au+Au (left) and $\mathrm{p}+\mathrm{p}$ (right) collisions. Despite using a smaller data sample and finer $p_{T}$ bins, the HFT results have significantly better precision than the published ones. The new results confirm the strong suppression of $D^{0}$ yields (relative to $\mathrm{p}+\mathrm{p}$ ) at high transverse momenta, which is comparable to the suppression observed for light hadrons, an indication of strong charm-medium interactions.

Leveraging the HFT also enables the first measurement of the second-order azimuthal anisotropy $\left(v_{2}\right)$ 


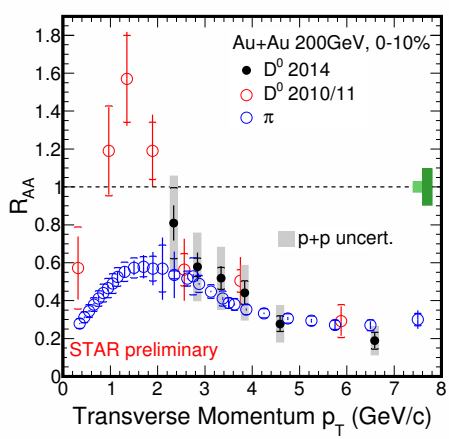

(a)

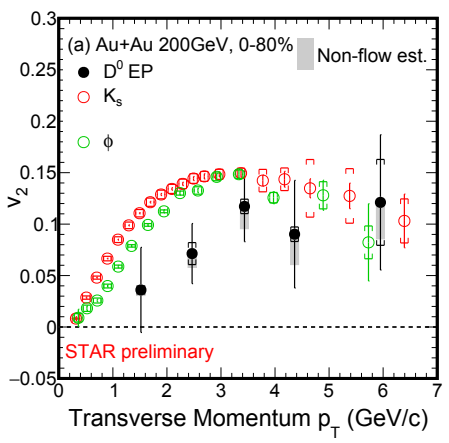

(b)

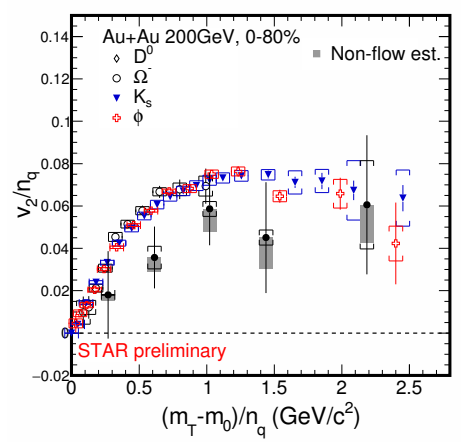

(c)

Figure 1: a) Measured $D^{0}$ nuclear modification factor, $R_{A A}$, with and without the HFT, compared to that of pions. b) Measured $D^{0} v_{2}$ compared to that of light hadrons. c) $v_{2} / n_{q}$ vs. $\left(m_{T}-m_{0}\right) / n_{q}$ for $D^{0}$ and other particle species.

for $D^{0}$ at RHIC. Figure $1 \mathrm{~b}$ shows the $D^{0} v_{2}$ in $0-80 \% \mathrm{Au}+\mathrm{Au}$ collisions which are significantly above zero for $p_{T}>2 \mathrm{GeV} / \mathrm{c}$ suggesting charm quarks flow with the medium. The vertical bars (brackets) represent statistical (systematic) uncertainties, while the grey shaded areas correspond to the estimate of contributions from non-flow effects. The rightmost panel, figure $1 \mathrm{c}$, shows $v_{2} / n_{q}$ as a function of $\left(\sqrt{m_{0}^{2}+p_{T}^{2}}-m_{0}\right) / n_{q}$, where $m_{0}$ is the hadron mass and $n_{q}$ the number of constituent quarks. The $D^{0}$ $v_{2} / n_{q}$ is systematically below those of light hadrons [5], but still consistent within uncertainties.

Figures $2 \mathrm{a}$ and $2 \mathrm{~b}$ show the comparison between the $D^{0} R_{A A}$ and $v_{2}$ to three theoretical models. The TAMU model [6] uses a non-perturbative T-matrix approach assuming that the two-body interactions can be described by a potential as a function of the transferred 4-momentum. In the case of $v_{2}$, two curves from TAMU are shown: the scenario including charm diffusion (blue) agrees with the data while the predictions without charm diffusion (magenta) are systematically lower. The calculation by the SUBATECH group [7] employs pQCD with Hard Thermal Loop approximation for soft collisions and can also describe both measurements over the whole $p_{T}$ range. In the model developed by the Duke group [12] the diffusion coefficient $2 \pi T D_{s}$ is a free parameter which, in the case of the red curve shown, has been constrained using the $R_{A A}$ measured at LHC to be roughly 7 . While both the SUBATECH and TAMU models show good agreement with the data in both observables, the calculation from the DUKE group under-predicts the $v_{2}$ observed in our data. The third panel, figure $2 \mathrm{c}$, shows the extracted diffusion coefficient from different model calculations compared to the yellow band on the far right, which indicates the range of inferred values that are compatible with the measurements presented here. It is worth noting that lattice QCD predictions [13], within large uncertainties, are consistent with the inferred range from data.

\section{Quarkonium production}

While the heavy quark production can be calculated by pQCD, how they evolve into quarkonia, a bound quark-antiquark pair, is intrinsically non-perturbative. For this reason, precise measurements of quarkonium production in $\mathrm{p}+\mathrm{p}$ collisions can help understand quarkonium production mechanisms. 


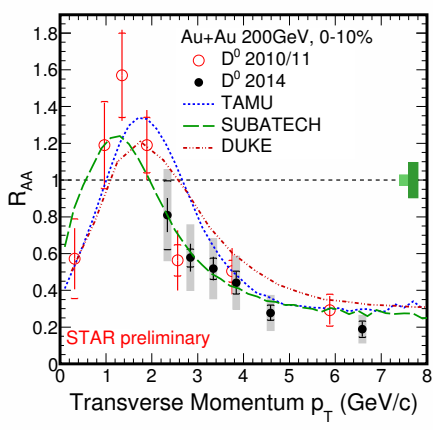

(a)

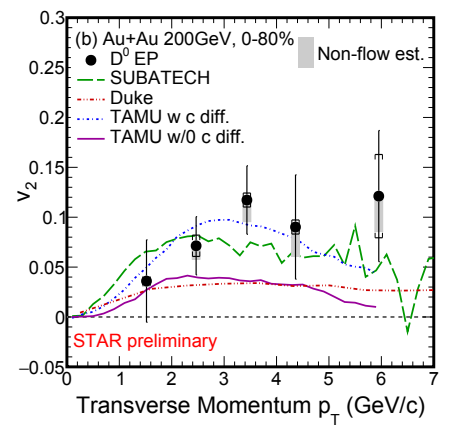

(b)

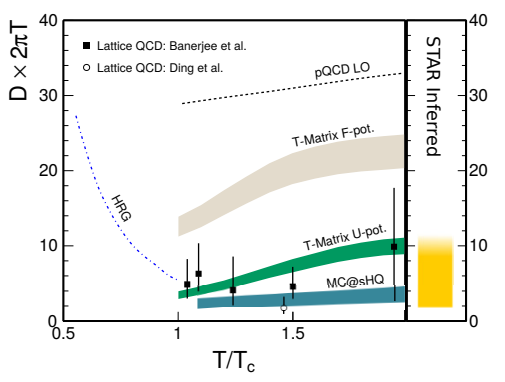

(c)

Figure 2: a) $D^{0} R_{A A}$ in $0-10 \%$ centrality compared to model calculations. b) $D^{0} v_{2}$ in $0-80 \%$ centrality compared to model calculations. c) Diffusion coefficient from model calculations and the inferred range from STAR measurements.

On the other hand, in heavy-ion collisions quarkonia may dissociate due to the color screening of the heavy quark-antiquark potential by the QGP. This dissociation should show dependence on the temperature of the QGP, and the binding energies of the different quarkonium states. Therefore the comparison between quarkonium production in heavy-ion collisions relative to $p+p$ collisions can be used to experimentally deduce the temperature of the QGP medium once the color screening, regeneration, and cold nuclear matter effects are fully understood. Below, we present $J / \psi$ and $\Upsilon$ measurements at STAR using the newly installed MTD described in previous sections.

\section{$4.1 \mathrm{~J} / \psi$ production}

$J / \psi$ invariant yields in $\mathrm{Au}+\mathrm{Au}$ collisions at $\sqrt{\mathrm{s}_{\mathrm{NN}}}=200 \mathrm{GeV}$ have been determined via the dimuon channel using the data recorded in 2014. The results (solid points) are shown as a function of the transverse momenta for different centrality classes in figure $3 \mathrm{a}$, which are seen to be consistent with the STAR published results in the dielectron channel (open points). Figure $3 \mathrm{~b}(3 \mathrm{c})$ shows the $R_{A A}$ for $p_{T}>0(5) \mathrm{GeV} / \mathrm{c}$ determined from the above mentioned MTD data (red stars) compared to the results from PHENIX [9] for $\mathrm{Au}+\mathrm{Au}$ collisions at $\sqrt{\mathrm{s}_{\mathrm{NN}}}=200 \mathrm{GeV}$ (black open circles) as well as the measurements from ALICE and CMS in $\mathrm{Pb}+\mathrm{Pb}$ collisions at $\sqrt{\mathrm{s}_{\mathrm{NN}}}=2.76 \mathrm{TeV}$ (blue squares). For $p_{T}>0 \mathrm{GeV} / \mathrm{c}$, the STAR results are consistent with the PHENIX results showing a decreasing trend towards more central collisions. On the other hand the ALICE results are almost independent of the centrality class and are significantly higher than those at RHIC in the central collisions. This difference could be due to a larger contribution from regeneration at low $p_{T}$ due to higher $c \bar{c}$ yields, increasing $J / \psi$ production at the LHC. At high $p_{T}$, the $R_{A A}$ at both RHIC and LHC have a decreasing trend towards more central collisions, and the LHC results are lower than those at RHIC. This is likely due to higher temperature reached at the LHC which leads to stronger $J / \psi$ dissociation. Two different transport models [10,11], both including dissociation and regeneration, can qualitatively describe the measured $R_{A A}$. There is, however, tension between the model calculations and experimental data at high transverse momenta. 


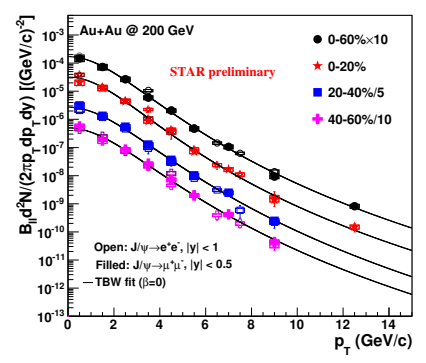

(a)

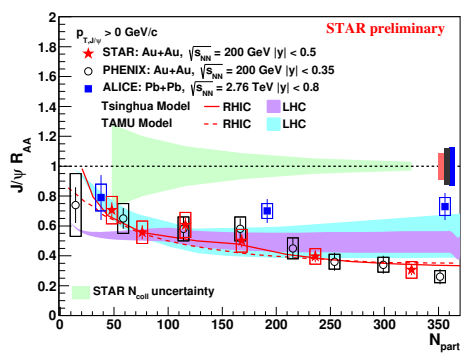

(b)

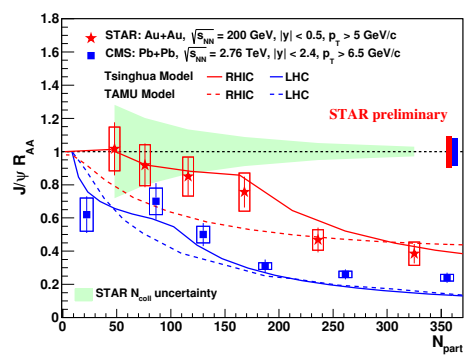

(c)

Figure 3: a) $J / \psi$ invariant yields in $\mathrm{Au}+\mathrm{Au}$ collisions at $\sqrt{\mathrm{S}_{\mathrm{NN}}}=200 \mathrm{GeV}$ in four centrality classes. b) $J / \psi R_{A A}$ vs. $N_{\text {part }}$ for $p_{T}>0 \mathrm{GeV} / \mathrm{c}$ in $\mathrm{Au}+\mathrm{Au}(\mathrm{Pb}+\mathrm{Pb})$ at $\sqrt{\mathrm{s}_{\mathrm{NN}}}=200 \mathrm{GeV}(2.76 \mathrm{TeV})$. c) $J / \psi$ $R_{A A}$ vs. $N_{\text {part }}$ for $p_{T}>5 \mathrm{GeV} / \mathrm{c}$ in $\mathrm{Au}+\mathrm{Au}(\mathrm{Pb}+\mathrm{Pb})$ at $\sqrt{\mathrm{s}_{\mathrm{NN}}}=200 \mathrm{GeV}(2.76 \mathrm{TeV})$.

\section{$4.2 \Upsilon$ production}

Using the MTD data described above, STAR has studied $\Upsilon$ production in Au+Au collisions at $\sqrt{\mathrm{S}_{\mathrm{NN}}}=200 \mathrm{GeV}$. Compared to the dielectron channel, the dimuon channel has the advantage of better invariant mass resolution due to the significantly suppressed bremsstrahlung radiation. Figure 4a shows the invariant mass spectrum of dimuon pairs. The unlike-sign (red points) distribution is fitted to the sum of $\Upsilon(1 S), \Upsilon(2 S)$ and $\Upsilon(3 S)$ signal distributions, $B \bar{B}$, Drell-Yan and the combinatorial background distributions. The shapes of the signal distributions are Gaussian functions with means fixed to the PDG values, and widths determined from detector simulations, while the shape of $B \bar{B}$ and Drell-Yan background is determined from PYTHIA. Both the shape and normalization of the combinatorial background are obtained by fitting the like-sign (black points) spectrum. Shown in figure $4 \mathrm{~b}$ are the ratio of the excited state yield to the ground state yield, obtained from the previous fit, together with the world-wide $\mathrm{p}+\mathrm{p}$ data and CMS measurements $[12,14]$ in $\mathrm{Pb}+\mathrm{Pb}$ collisions at $\sqrt{\mathrm{s}_{\mathrm{NN}}}=2.76$ $\mathrm{TeV}$. There is an indication of larger $\Upsilon(2 S+3 S) / \Upsilon(1 S)$ ratio at RHIC than that at the LHC.

\section{Summary}

STAR has carried out the first heavy flavor measurements in heavy-ion collisions using the newly installed HFT and MTD detectors. The measured charmed meson $v_{2}$ in Au+Au collisions is found to be non-zero, but systematically below the $v_{2}$ of light hadrons in 0-80\% centrality, while the measurement of the $R_{A A}$ using the HFT shows a similar level of suppression for the charmed meson as the light hadrons at high transverse momenta. Comparison to a series of models shows that they are able to describe both the $v_{2}$ and $R_{A A}$ simultaneously, favouring a scenario where charm quarks flow with the medium. These comparisons can be used to infer a range, between 2 and $\sim 12$, for the value of the charm diffusion coefficient $2 \pi T D_{s}$.

The measurements of $J / \psi$ with the MTD via the dimuon channel yield consistent results as obtained through the dielectron channel and show a distinct rising trend in the $R_{A A}$ with transverse momenta in $20-60 \%$ centrality. The suppression observed at high $p_{T}$ in $0-20 \%$ central collisions is consistent with dissociation due to color screening. 


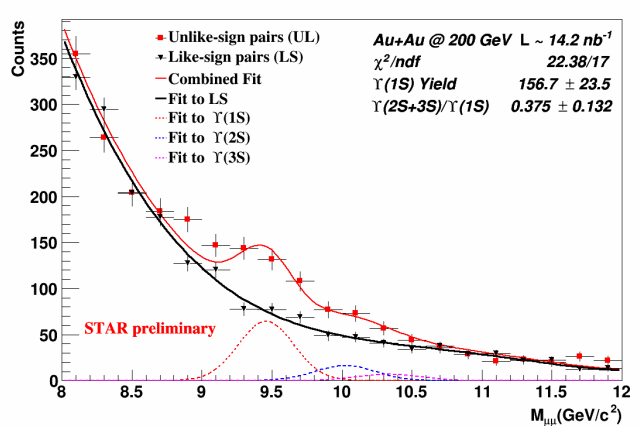

(a)

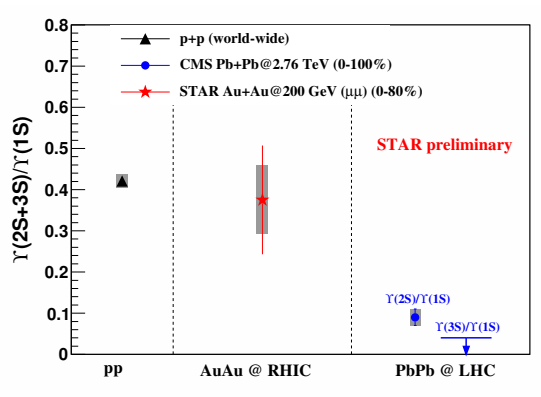

(b)

Figure 4: a) Invariant mass distribution of dimuon pairs in $\mathrm{Au}+\mathrm{Au}$ collisions at $\sqrt{\mathrm{S}_{\mathrm{NN}}}=200 \mathrm{GeV}$. b) Ratio of excited to ground $\Upsilon$ states from world data in $\mathrm{p}+\mathrm{p}$ collisions, Au+Au collisions at $\sqrt{\mathrm{s}_{\mathrm{NN}}}=$ $200 \mathrm{GeV}$ and $\mathrm{Pb}+\mathrm{Pb}$ collisions at $\sqrt{\mathrm{s}_{\mathrm{NN}}}=2.76 \mathrm{TeV}$.

\section{Acknowledgements}

This material is based upon work supported by the U.S. Department of Energy, Office of Science, Office of Workforce Development for Teachers and Scientists, Office of Science Graduate Student Research (SCGSR) program. The SCGSR program is administered by the Oak Ridge Institute for Science and Education for the DOE under contract number DE-AC05-06OR23100.

\section{References}

[1] L. Adamczyk et al. (STAR Collaboration), Phys. Rev. Lett. 113 (2014) 142301.

[2] L. Adamczyk et al. (STAR Collaboration), arXiv 1607.07517 (2016).

[3] Technical Design Report: The STAR Heavy Flavor Tracker (2011).

[4] L. Ruan et al., J. Phys. G: Nucl. Part. Phys. 36 (2009) 095001.

[5] B. I. Abelev et al. (STAR Collaboration), Phys. Rev. C 77(5) (2008) 054901

[6] M. He, R. J. Fries and R. Rapp, Phys. Rev. C 86(1) (2012) 014903

[7] M. Nahrgang et. al., Phys. Rev. C 91(1) (2015) 014904

[8] Cao S, Qin G Y and Bass S A, Phys. Rev. C 88(4) (2013) 044907

[9] A. Adare et al. (PHENIX collaboration), Phys. Rev. D 82 (2010) 012001.

[10] Y. Liu et. al. , Phys. Let. B 678 (2009) 72; K. Zhou et. al. , Phys. Rev. C 89 (2014) 054911.

[11] X. Zhao and R. Rapp, Phys. Rev. C 82 (2010) 064905.

[12] W. Zha et. al., Phys. Rev. C 88 (2013) 067901

[13] A. Andronic et. al., The Eur. Phys. J. C 76 (2016) 3107

[14] S. Chatrchyan et al. (CMS Collaboration), PRL 109 (2012) 222301 